\title{
Energetic and Structural Analysis of the Role of Tryptophan 59 in FKBP12
}

\author{
Kate F. Fulton, Sophie E. Jackson, and Ashley M. Buckle*,† \\ MRC Centre for Protein Engineering, Hills Road, Cambridge, CB2 2QH, U.K., and \\ Cambridge University Chemical Laboratory, Lensfield Rd, Cambridge, CB2 IEW, U.K.
}

Received September 3, 2002; Revised Manuscript Received November 12, 2002

\begin{abstract}
Tryptophan 59 forms the seat of the hydrophobic ligand-binding site in the small immunophilin FKBP12. Mutating this residue to phenylalanine or leucine stabilizes the protein by 2.72 and $2.35 \mathrm{kcal}$ $\mathrm{mol}^{-1}$, respectively. Here we report the stability data and $1.7 \AA$ resolution crystal structures of both mutant proteins, complexed with the immunosuppressant rapamycin. Both structures show a relatively large response to mutation involving a helical bulge at the mutation site and the loss of a hydrogen bond that anchors a nearby loop. The increased stability of the mutants is probably due to a combination of improved packing and an entropic gain at the mutation site. The structures are almost identical to that of wild-type FKBP12.6, an isoform of FKBP12 that differs by 18 residues, including Trp59, in its sequence. Therefore, the structural difference between the two isoforms can be attributed almost entirely to the identity of residue 59. It is likely that in FKBP12-ligand complexes Trp59 provides added binding energy at the active site at the expense of protein stability, a characteristic common to other proteins. FKBP12 associates with the ryanodine receptor in skeletal muscle (RyR1), while FKBP12.6 selectively binds the ryanodine receptor in cardiac muscle (RyR2). The structural response to mutation suggests that residue 59 contributes to the specificity of binding between FKBP12 isoforms and ryanodine receptors.
\end{abstract}

Human FKBP12, a peptidyl-prolyl isomerase that binds the immunosuppresant drugs FK506 and rapamycin, is a ubiquitous cytosolic protein implicated in several physiological processes; yet its mechanism of action is not fully understood (Schreiber, 1991; Harding et al., 1989; DeCenzo et al., 1996; Harrar et al., 2001). FKBP12-FK506/rapamycin complexes inhibit key events in the cytoplasmic signal process, leading to immunosuppression (Schreiber, 1991). FKBP12 and its isoform FKBP12.6 have been found to be crucial regulators of intracellular $\mathrm{Ca}^{2+}$ release due to their association with ryanodine receptors, thus playing essential roles in excitation-contraction coupling in skeletal and cardiac muscle (Jayaraman et al., 1992; Brillantes et al., 1994; Xin et al., 1999; Carmody et al., 2001; Gaburjakova et al., 2001). Disruption of the FKBP12.6 gene in mice results in cardiac hypertrophy (Xin et al., 2002), while developmental cardiac defects have been reported in FKBP12-deficient mice (Shou et al., 1998). Despite normal skeletal muscle, the mutant mice compared with wild type were observed to have both RyR1 and RyR2 $\mathrm{Ca}^{2+}$ channels in more frequent open-states. The results of Shou et al., (1998) are consistent with the finding that paediatric organ transplant patients given high doses of FK506 developed hypertrophic cardiomyopathy associated with clinically severe heart failure (Atkinson, 1995). Further, genetically transmitted mutations in the hRyR 2 gene causes stress-induced bidirectional ventricular tachycardia (Laitinen et al., 2001; Priori et al., 2001), leading to cardiac arrest and sudden death. It has been suggested that these mutations disrupt the FKBP12.6 association, leaving RyR2 in an open state (Marks, 2002).

\footnotetext{
* To whom correspondence should be addressed.

† Present address: Department of Biochemistry and Molecular Biology, School of Biomedical Sciences, Faculty of Medicine, Monash University, Clayton, Vic 3168, Australia.
}

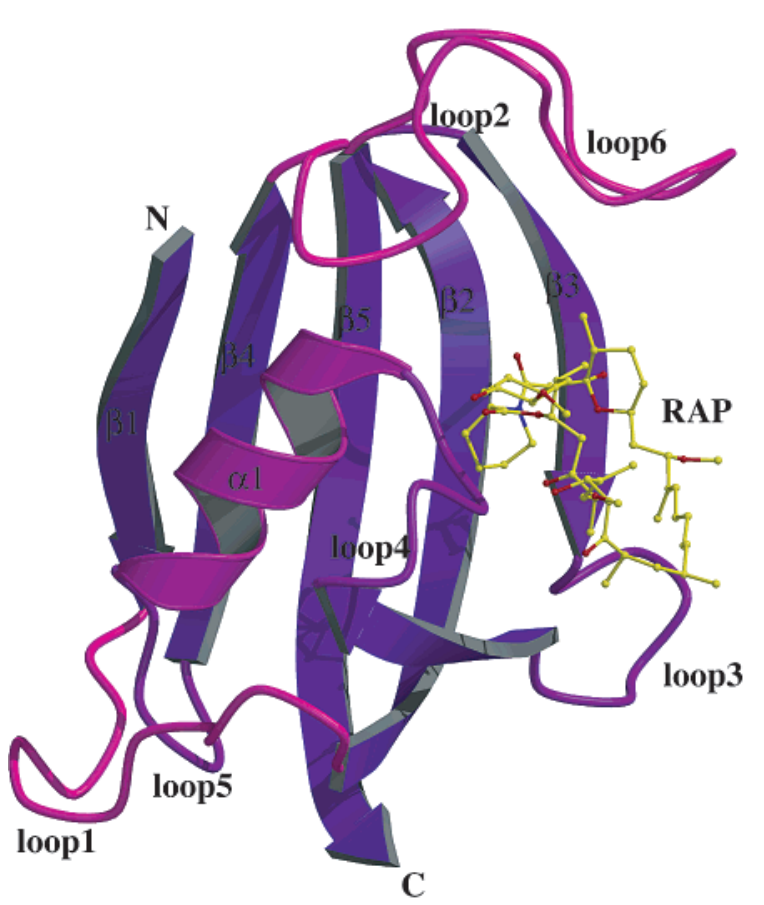

FIGURE 1: The structure of human FKBP12. The secondary structure representation was produced using MolScript (Kraulis, 1991) and rendered using Raster3D (Merrit and Murphy, 1994).

Owing to its small size ( $12 \mathrm{kDa})$ and its two-state folding behavior, FKBP12 is a good model system for protein folding and engineering studies (Main et al., 1999). Its structure, in free form and complexed with FK506 and rapamycin, has been solved by X-ray crystallography to high resolution (Michnick et al., 1991; van Duyne et al., 1991a, 1991b, 1993) (Figure 1). Its folding pathway and stability have been studied extensively (Main et al., 1998, 1999a, 1999b; Fulton et al., 
1999; Main et al., 2001). FKBP12.6, an isoform of FKBP12, differs in its sequence by 18 out of 108 residues (83\% sequence identity; Timerman et al., 1996). One notable difference is residue 59, which forms the seat of the drug binding site and which is a tryptophan in FKBP12 and a phenylalanine in FKBP12.6. To explore the role of Trp59 in FKBP12, we have investigated the structural and energetic consequences of mutating this residue to phenylalanine and leucine. Both mutations produced proteins with increased stability. We describe their structures, determined by X-ray crystallography at $1.7 \AA$ A resolution, and provide a structural rationalization for the stabilization. We then discuss the structural response to mutation in the context of the variation in binding specificity between FKBP12/FKBP12.6 and ryanodine receptors.

\section{MATERIALS AND METHODS}

Expression and Purification of the Wild-Type and Mutants. Wild-type and mutant FKBP12 were constructed, expressed, and purified as described (Main et al., 1998). Protein concentration was determined spectrophotometrically using a molar extinction coefficient, $\epsilon$, of 9927 and $4243 \mathrm{M}^{-1} \mathrm{~cm}^{-1}$ at $278 \mathrm{~nm}$ for wild-type and mutant FKBP12, respectively. Ultra-grade urea was used (Fisher Scientific UK Ltd.). All stock solutions of urea were made using volumetric flasks, flash-frozen, and stored at $20 \mathrm{deg} \mathrm{C}$ to prevent degradation. In all experiments the final buffer concentrations were 50 $\mathrm{mM}$ Tris- $\mathrm{HCl}$ (pH 7.5), 1 mM DTT. All other materials were analytical grade and purchased from Sigma.

Equilibrium Unfolding. Unfolding experiments under equilibrium conditions to measure the relative stabilities of wild type, WF59, and WL59 were performed and analyzed as described elsewhere (Main et al., 1998). For the mutants, an excitation wavelength of $280 \mathrm{~nm}$ was used and the emission at $303 \mathrm{~nm}$ recorded.

Structure Solution and Refinement of Wild-Type and Mutant FKBP12-Rapamycin Complexes. The structure of wild-type FKBP12, both in free forms and complexed with rapamycin and FK506, is known from both crystallographic and solution studies (Michnick et al., 1991; van Duyne et al., 1991, 1993; Moore et al., 1991). All published structures were derived from experiments conducted at $298 \mathrm{~K}$. Data for the mutants WF59 and WL59 were collected from cryocooled crystals at $100 \mathrm{~K}$. To eliminate differences due to the temperature of data collection, the structure of the wildtype protein was also determined from data collected at $100 \mathrm{~K}$.

Crystals of wild-type FKBP12 and mutants WL59 and WF59, complexed with rapamycin, were obtained by hanging drop vapor diffusion (McPherson, 1982), in 30\% PEG MME $2 \mathrm{~K}, 0.2 \mathrm{M}\left(\mathrm{NH}_{4}\right)_{2} \mathrm{SO}_{4}(\mathrm{pH} 4.6)$ and belong to spacegroup $P 2{ }_{1} 2_{1} 2_{1}$. In all three cases, a complete dataset to $1.7 \AA$ resolution was collected from a single crystal cooled to 100 $\mathrm{K}$ using a $345 \mathrm{~mm}$ Mar Research image plate detector, with in-house monochromatic $\mathrm{Cu} \mathrm{K} \alpha \mathrm{X}$-rays generated by a Elliot GX13 rotating anode. This crystal form is isomorphous with that of the published FKBP12-rapamycin complex (van Duyne et al., 1991a, 1991b, 1993), with the notable distinction that for both mutants there is a $3.4 \AA$ reduction in the unit cell $c$ axis (due to a rigid body shift of the protein molecule: see below). This is a direct result of the structural
Table 1: Thermodynamic Parameters for the Reversible Unfolding of Wild-Type and Mutant FKBP12

\begin{tabular}{lccc}
\hline \multicolumn{1}{c}{ mutant } & $\begin{array}{c}{\left[\text { urea }_{50 \%}{ }^{a}\right.} \\
(\mathrm{M})\end{array}$ & $\begin{array}{c}m_{\mathrm{D}-\mathrm{N}}{ }^{a} \\
\left(\mathrm{kcal} \mathrm{mol}^{-1} \mathrm{M}^{-1}\right)\end{array}$ & $\begin{array}{c}{ }^{b} \Delta \Delta G_{\mathrm{D}-\mathrm{N}} \\
\left(\mathrm{kcal} \mathrm{mol}^{-1}\right)\end{array}$ \\
\hline wild type & $3.87 \pm 0.02$ & $1.43 \pm 0.05$ & 0 \\
WF59 & $5.58 \pm 0.20$ & $1.11 \pm 0.16$ & $-2.72 \pm 0.32$ \\
WL59 & $5.35 \pm 0.05$ & $1.20 \pm 0.08$ & $-2.35 \pm 0.09$ \\
\hline
\end{tabular}

${ }^{a}$ Errors are from the data fitting. ${ }^{b}$ Calculated using an average $m_{\mathrm{D}-\mathrm{N}}$ value of $1.59 \pm 0.02 \mathrm{kcal} \mathrm{mol}^{-1} \mathrm{M}^{-1}$ calculated from data for wild type and a large number of mutants (Main et al., 1998). A negative sign indicates that the mutant is more stable than the wild-type protein.

Table 2: Results of Crystallographic Data Processing and Structure Refinement Statistics ${ }^{a}$

\begin{tabular}{|c|c|c|c|}
\hline FKBP12 & WT & WF59 & WL59 \\
\hline \multicolumn{4}{|c|}{ Data Collection } \\
\hline space group & $P 2{ }_{1} 2_{1} 2_{1}$ & $P 2_{1} 2_{1} 2_{1}$ & $P 2_{1} 2_{1} 2_{1}$ \\
\hline $\begin{array}{l}\text { cell dimensions }(\AA) \text { : } \\
\quad a, b, c\end{array}$ & $44.6,48.8,54.0$ & $44.5,49.1,51.7$ & $44.1,49.4,51.6$ \\
\hline resolution $(\AA)$ & $22.4-1.7$ & $20.4-1.7$ & $20.3-1.7$ \\
\hline$R_{\text {merge }}^{b}$ & $5.6(15.6)$ & $6.4(17.8)$ & $5.2(27.2)$ \\
\hline completeness (\%) & $96.1(91.9)$ & $96.2(95.9)$ & $89.2(91.2)$ \\
\hline multiplicity & $6.4(6.4)$ & $3.7(3.5)$ & $3.7(3.3)$ \\
\hline$\langle I / \sigma I\rangle$ & $39.2(7.0)$ & $23.7(3.8)$ & $20.3(2.6)$ \\
\hline \multicolumn{4}{|c|}{ Structure Refinement } \\
\hline$R_{\text {free }}(\%)^{c}$ & 25.8 & 22.9 & 27.0 \\
\hline$R_{\text {cryst }}(\%)$ & 21.8 & 19.0 & 20.9 \\
\hline$\Delta_{\text {bond }}(\AA)$ & 0.008 & 0.008 & 0.009 \\
\hline$\Delta_{\text {angle }}(\operatorname{deg})$ & 1.7 & 1.7 & 2.0 \\
\hline mean $B\left(\AA^{2}\right)$ & $15.2(13.7)^{a}$ & $18.6(17.3)^{a}$ & $25.3(25.1)^{a}$ \\
\hline $\begin{array}{l}\text { no. of solvent } \\
\text { molecules in model }\end{array}$ & 165 & 144 & 145 \\
\hline $\begin{array}{l}\text { no. of glycerol } \\
\text { molecules in model }\end{array}$ & 1 & 1 & 2 \\
\hline
\end{tabular}

${ }^{a}$ Figure in parentheses refer to the highest-resolution shell. ${ }^{b}$ Agreement between intensities of repeated measurements of the same reflections and can be defined as: $\sum\left(I_{h, i}-\left\langle I_{h}\right\rangle\right) / \sum I_{h, i}$, where $I_{h, i}$ are individual values and $\left\langle I_{h}\right\rangle$ is the mean value of the intensity of reflection $h$. $^{c}$ The free $R$ factor was calculated with the $10 \%$ of data omitted from the refinement.

response to mutation, discussed below. It is also noteworthy that cooling to $100 \mathrm{~K}$ does not cause significant changes to the unit cell volume.

Datasets were indexed and the reflection intensities measured using MOSFLM (CCP4, 1994). The CCP4 suite was used for data reduction (CCP4, 1994). Five percent of the dataset for each mutant was flagged for calculation of the free $R$ factor (Brunger, 1992, 1993) during refinement. The same reflections were flagged for all structures to minimize coupling of reflections between calculated and observed structure factors (Kleywegt and Brunger, 1996). Structures were solved using the wild-type FKBP12-rapamycin complex (PDB code 1fkb, van Duyne et al., 1991a, 1991b, 1993) as a starting model for rigid-body refinement. Iterative cycles of maximum likelihood refinement using REFMAC (Murshudov et al., 1997) and model building in O (Jones et al., 1991) were used to further improve the model, until there was no further drop in free $R$-factor. All structures consist of 107 protein residues corresponding to the full-length protein.

\section{RESULTS}

Equilibrium unfolding data are shown in Table 1. For both mutants a large stabilization is observed. For Trp $\rightarrow$ Phe59, 

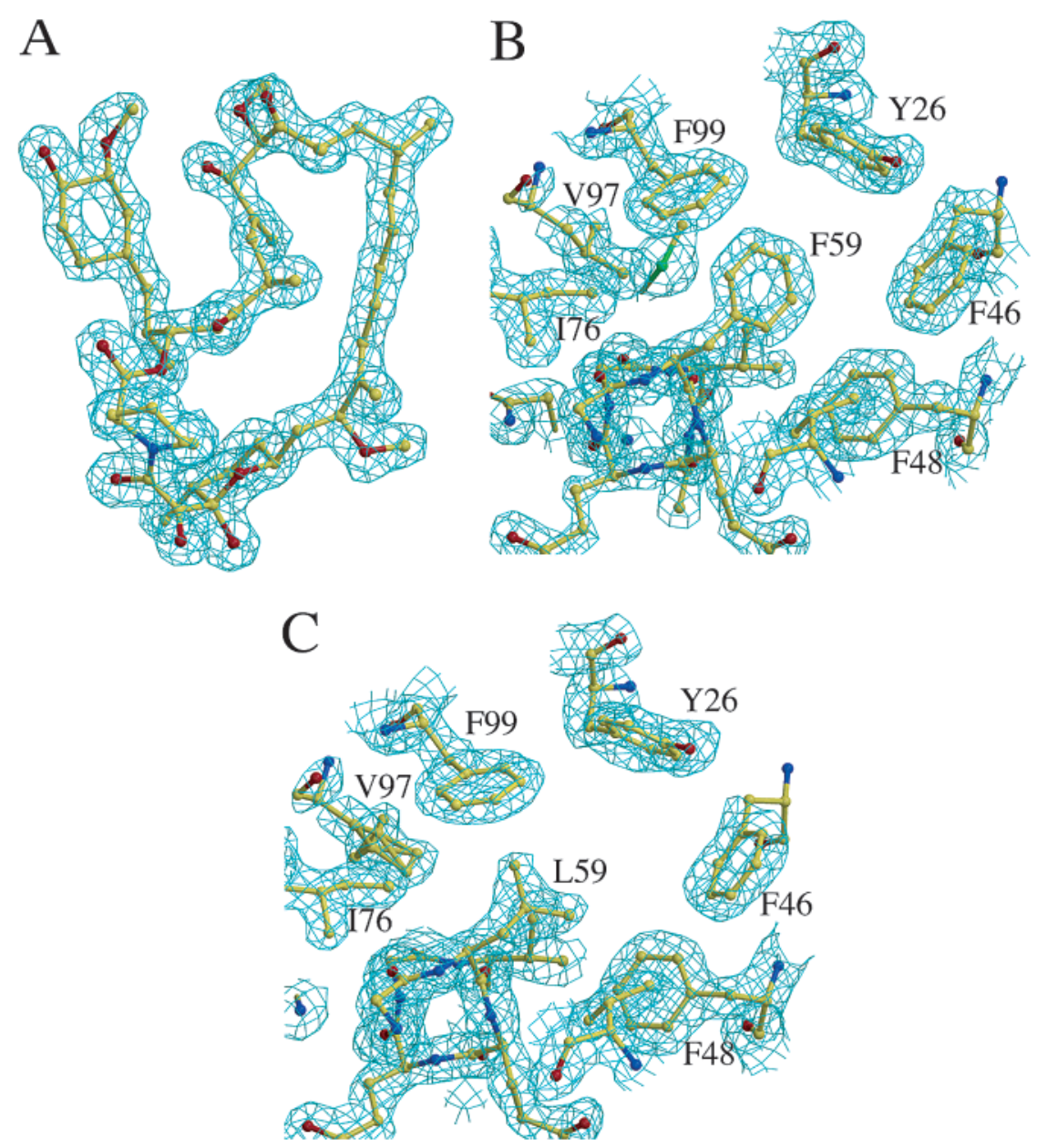

FIGURE 2: $2 F_{\mathrm{o}}-F_{\mathrm{c}}$ electron density maps. Calculated with SIGMAA coefficients (Read, 1986), contoured at $1 \sigma$ ( $\sigma$ is the root-mean-square deviation from the mean electron density in the unit cell). Superimposed is the refined atomic model. Drawn with BobScript (extensions to the program MolScript (Kraulis, 1991), Robert Esnouf), and Raster3D (Merrit and Murphy, 1994). (a) Rapamycin molecule in WF59; (b) Phe59 and surrounding residues in WF59, looking down $\alpha$-helix from the N-terminus, away from rapamycin binding site; (c) Leu59 and surrounding residues in WL59, looking down $\alpha$-helix from the N-terminus, away from rapamycin binding site.

$\Delta \Delta G_{\mathrm{D}-\mathrm{N}}=-2.72 \mathrm{kcal} \mathrm{mol}^{-1}$, and for Trp $\rightarrow$ Leu59 $\Delta \Delta G_{\mathrm{D}-\mathrm{N}}=-2.35 \mathrm{kcal} \mathrm{mol}^{-1}$. In general, removal of such a large number of methyl(ene) groups from the hydrophobic core is predicted to result in a large destabilization of the protein. This was seen in the case of barnase, where tryptophan mutations resulted in a large destabilization of the protein: $\Delta \Delta G_{\mathrm{D}-\mathrm{N}}=1.52 \mathrm{kcal} \mathrm{mol}^{-1}(\mathrm{Trp} \rightarrow$ Leu94) and $\Delta \Delta G_{\mathrm{D}-\mathrm{N}}=0.87 \mathrm{kcal} \mathrm{mol}^{-1}(\mathrm{Trp} \rightarrow$ Phe94) (Alan Fersht, personal communication, 2002).

Crystallographic data are summarized in Table 2. The quality of the electron density in all models is excellent throughout. Overall, the models have excellent stereochemistry. On the basis of a stereochemical analysis with the program PROCHECK (Morriset et al., 1992), 90\% of residues have backbone $\phi / \psi$ angles within the most favored regions of the Ramachandran plot. No residues have disallowed backbone $\phi / \psi$ angles. The main-chain and side-chain stereochemistry of the model is better than that derived from the 118 protein structures solved at $2.0 \AA$ resolution used in the PROCHECK analysis. Several residues in all three models have clearly defined multiple side-chain conformations (see Table 2). Disordered atoms in these residues were given fractional occupancies and included in the refinement.

One or more molecules of glycerol could be built into the electron density in all cases. These molecules, which had diffused into the crystals during the very brief $(<5 \mathrm{~s})$ period of soaking in the cryoprotectant, are involved in hydrogen bonding interactions with the protein surface. The electron density around residues 90 and 98 , which are discretely disordered in the wild-type structure at 298 K (van Duyne et al., 1993), is very well defined for wild-type and mutant structures at $100 \mathrm{~K}$, with no indication of more than one conformation. It is likely that the low temperature has "frozen" the conformation of both of these relatively solvent exposed side chains, possibly via the ordering of surrounding solvent. In all three cases, fragmented electron density is observed for additional N-terminal residues Gly-Ser-Met which are the result of the cloning and expression in pRSETA-His tag vector. However, it was impossible to model these residues into the electron density.

The Structural Response to the Mutations WF59 and WL59. The structures of wild-type, WF59 and WL59 FKBP12, complexed with rapamycin are shown in Figure 3. The rapamycin and FK506-binding site is a shallow hydrophobic cleft between the $\alpha$-helix and the five-stranded antiparallel $\beta$-sheet. The overall structures of WF59 and WL59 are very similar to the wild-type structure. A leastsquares superposition of backbones of WF59 and WL59 with wild type gives an average root-mean-square deviation (rmsd) of 0.35 and $0.38 \AA$ for all the main-chain atoms of WF59 


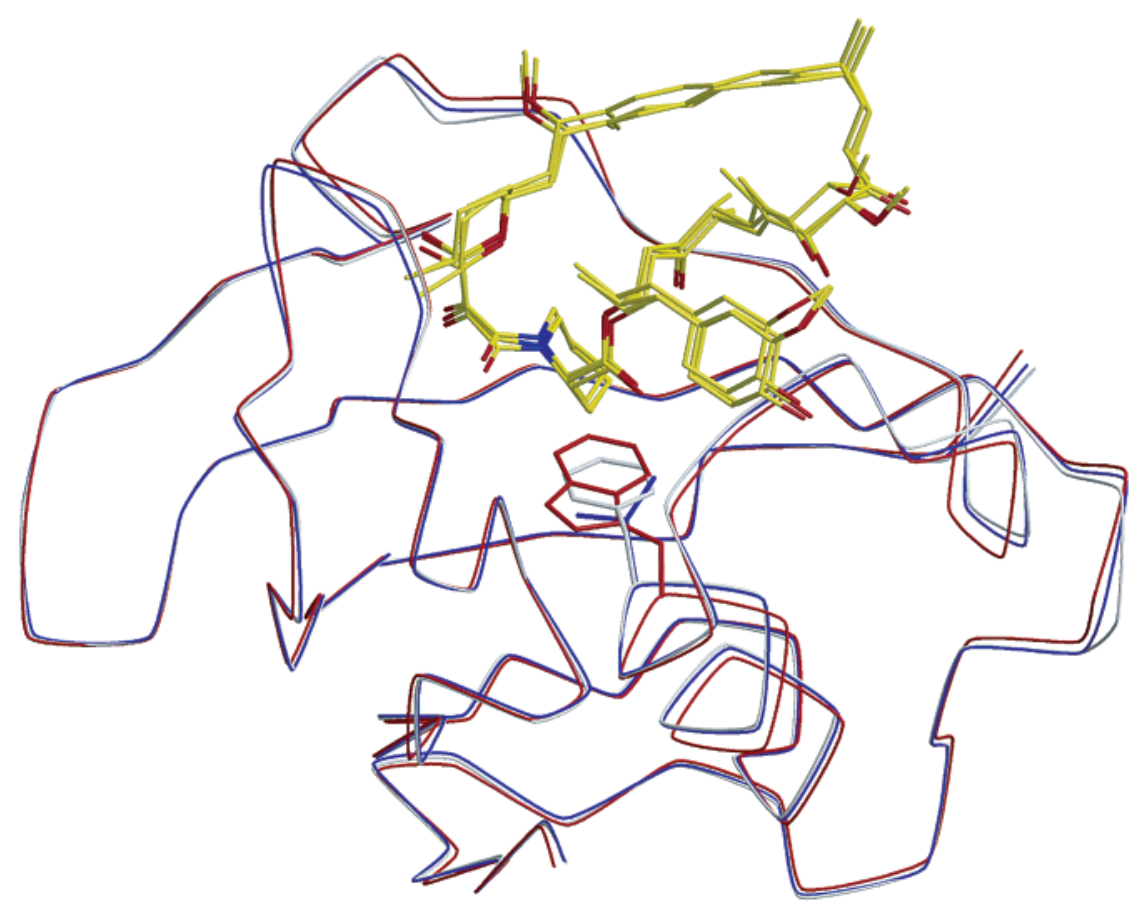

FIGURE 3: Structural overlay of wild-type (red), WF59 (white), and WL59 (blue) FKBP12, showing residue 59 and rapamycin. Drawn with MolScript (Kraulis, 1991) and Raster3D (Merrit and Murphy, 1994).

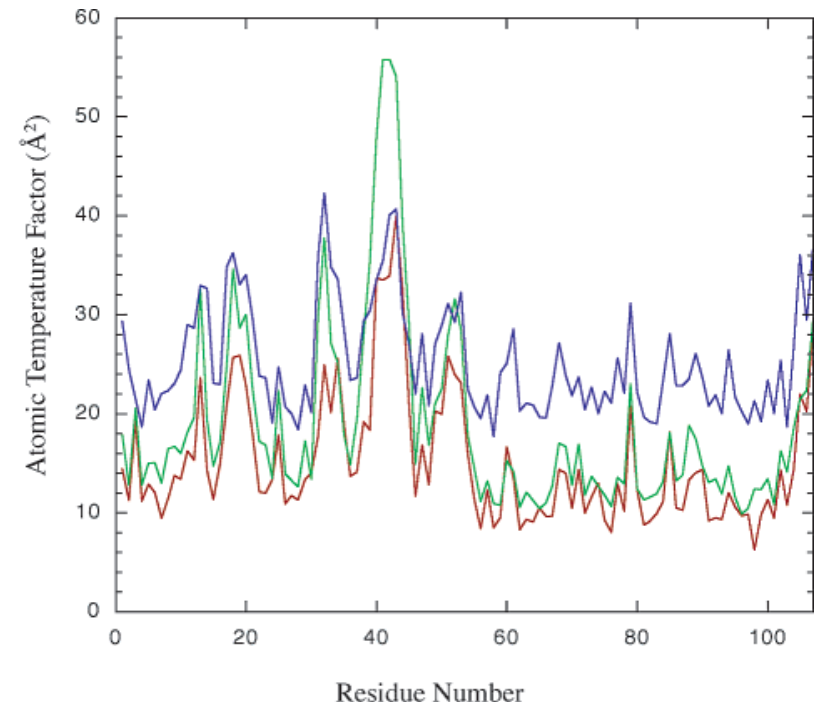

FIGURE 4: Plot of average atomic temperature $(B)$ factors for wildtype (red), WF59 (green), and WL59 (blue) structures.

and WL59, respectively. The rmsd values for side-chain atoms are somewhat larger, $0.52 \AA$ for WF59 and $0.48 \AA$ for WL59. The atomic temperature $(B)$ factors for each structure are shown and compared in Figure 4. The average $B$ factors for main-chain atoms, side-chain atoms, rapamycin atoms, and water are as follows: 13.9, 16.7, 13.7, and 29.3 $\AA^{2}$ for WT; 17.4, 20.0, 17.3, and $32.8 \AA^{2}$ for WF59; and 24.0, 26.8, 25.1, and $40.4 \AA^{2}$ for WL59, respectively.

There are some large deviations in the positions and conformations of surface side chains but these are mainly attributable to changes in crystal packing. The structural response to both mutations is restricted to the local structure surrounding Trp59 and can be described as follows. There are no significant movements in the $\beta$-sheet structure adjacent to the mutation site. In both structures the Leu59 and Phe59 side chains, which are extremely well resolved in the electron density (Figure 2), occupy similar volumes. Both have lowenergy, preferred conformations (Ponder and Richards, 1987). The $\alpha$-helix (56-66) has shifted inward toward the cavity created by the removal of the tryptophan side chain (Figures 3, 5, and 6). This is more pronounced at the $\mathrm{N}$-terminal end of the $\alpha$-helix $(58-62)$ (the shift in $\mathrm{C}_{\alpha}$ atoms is maximal at residue 59: $0.9 \AA$ for WF59 and $0.8 \AA$ for WL59). Since the shifts diminish further toward the Cterminus of the helix, the result is a "bulging" at the middle. Although the cavity created by removal of Trp59 cannot be filled by the smaller Phe side chain, Phe59 has moved toward the center of the cavity such that the center of its side chain is at approximately the same position as the center of the side chain of Trp59 in wild type. These shifts have important consequences for the loop region 50-54. It appears that the hydrogen bonds between Glu60 ( $\alpha$-helix) and the backbone amide NH groups of residue 51 and 53 (loop4) are important anchors for this loop. The helical "bulging" toward the mutation site disrupts the side chain of Glu60 and hence the hydrogen bond with the backbone nitrogen of residue 53 (Figures $5 \mathrm{~b}$ and $6 \mathrm{~b}$ ). In both mutant structures, the side chain of Glu60 adopts a different conformation and the hydrogen bond with the backbone nitrogen of residue 51 is retained, albeit with altered geometry. In summary, the conformation of this loop region is directly affected by the mutations.

There are no significant structural shifts of rapamycin atoms. Generally, the flexibility around the mutation site has increased in both mutants, as indicated by the comparison of atomic temperature factors (Figure 4) and by the increased number of discrete conformations that can be modeled for surrounding side chains (Table 3 ). In particular, the conformational disorder at residues 50 and 74 is of significant interest (Figure 7). This loop region 50-54 packs against the $\alpha$-helix, and the decrease in conformational order is 


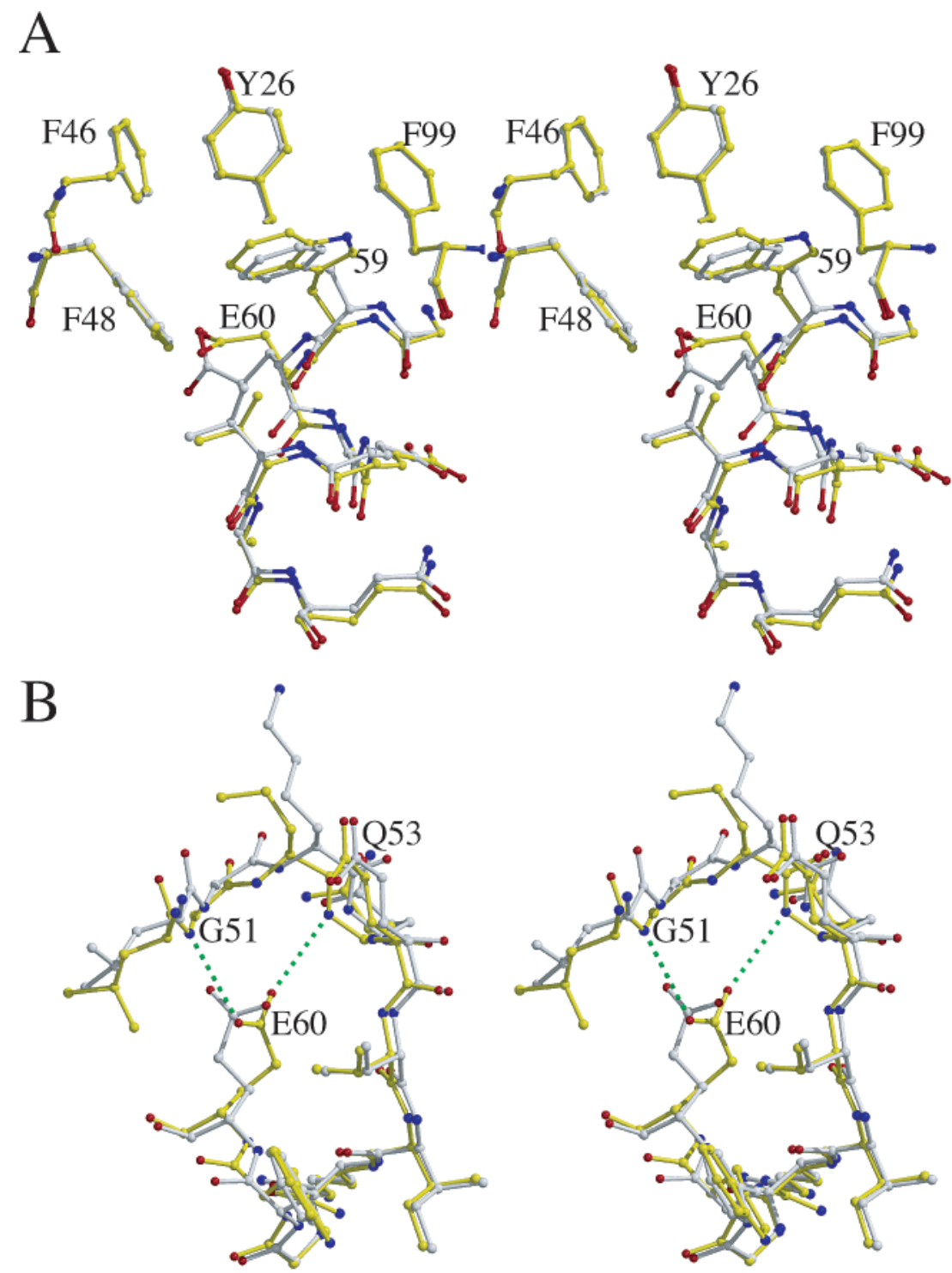

FIGURE 5: Superposition of the structures of WF59 (white bonds) and wild-type (yellow bonds) FKBP12 shown in stereo. Drawn with MolScript (Kraulis, 1991) and Raster3D (Merrit and Murphy, 1994): (a) $\alpha$-helix and residues surrounding mutation site; (b) loop4 region (residues 50-54) showing the hydrogen bonds between Glu60 ( $\alpha$-helix) and the backbone amide NH groups of residues 51 and 53.

strongly correlated to the inward helical movement described above. In the WL59 mutant, Leu74 adopts two discrete conformations, but in the wild-type and WF59 structures, only one (the same) conformation is modeled. Disorder of Leu74 in the case of WL59 is directly related to the conformational space created at residue 74 from the shifts at residue 59 (Figure $7 \mathrm{~b}$ ).

Structural Comparisons with FKBP12.6, an Isoform of $F K B P 12$. FKBP12.6 is an isoform of FKBP12, differing in sequence in only 18 out of 107 residues (Figure 8). Seven of these residues are polar or charged and are found on the surface. The remaining nine residues are hydrophobic and are found in the protein interior. Residue 59, located at the base of the rapamycin and FK506-binding sites, is a phenylanine in FKBP12.6 and a tryptophan in FKBP12. The structures of FKBP12 mutants are almost identical to that of wild-type FKBP12.6 (Figures 8 and 9) (Deivanayagam et al., 2000). Thus, the structural differences between FKBP12 and FKBP12.6 are due almost entirely to the difference at residue 59 .

\section{DISCUSSION}

Increased Stability of Mutants Suggests a Tradeoff between Stability and Function. Mutants WF59 and WL59 are 2.72 and $2.35 \mathrm{kcal} \mathrm{mol}^{-1}$ more stable than the wild-type protein. The gain in stability is very likely associated with improved packing as a result of the structural shifts. Indeed, structural shifts upon mutation must always occur to lower the free energy. There is no evidence of strain present in the wildtype structure, but it is possible that the decrease in order of the loop region 50-53 upon mutation could bring about a favorable entropic gain. Further stabilization in WL59 may be attributed to the disorder of Leu74. Mutagenesis studies on several proteins have shown that there is a compromise between stability and activity: residues at active sites are optimized for function, often at the expense of protein stability (Meiering et al., 1992). It is likely that this phenomenon is responsible for the stabilization observed here. Indeed, this is a proven method of increasing protein stability: using an empirical approach of exploring naturally occurring variations among a family of homologous proteins, 


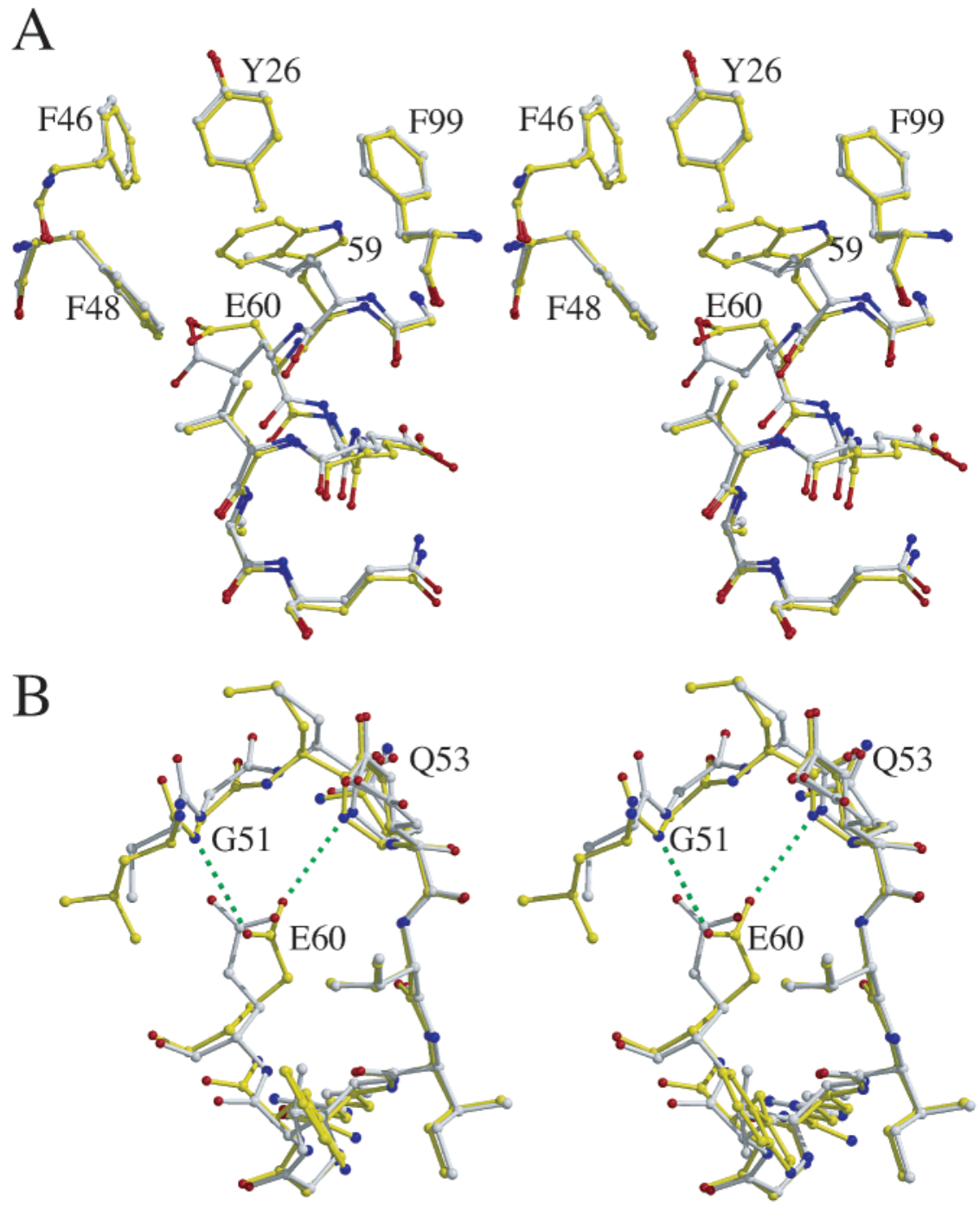

FIGURE 6: Superposition of the structures of WL59 (white bonds) and wild-type (yellow bonds) FKBP12 shown in stereo. Drawn with MolScript (Kraulis, 1991) and Raster3D (Merrit and Murphy, 1994): (a) $\alpha$-helix and residues surrounding mutation site; (b) loop4 region (residues 50-54) showing the hydrogen bonds between Glu60 ( $\alpha$-helix) and the backbone amide NH groups of residues 51 and 53 .

\begin{tabular}{lcccc}
\hline \multicolumn{4}{l}{ Table 3: Residues Having Multiple Conformations } \\
\hline $\begin{array}{c}\text { no. of discrete } \\
\text { conformations }\end{array}$ & WT & WF59 & WL59 & WT (298 K $)^{a}$ \\
\hline 2 & Ser77 & Ser38, Ser77 & $\begin{array}{l}\text { Met29, Ser38, } \\
\text { Glu54, Leu74 }\end{array}$ & Ile90, Va198 \\
3 & - & Leu50 $^{b}$ & $c$ & - \\
\hline
\end{tabular}

${ }^{a}$ van Duyne et al., 1993. ${ }^{b}$ The residues shown in bold are buried in the hydrophobic core of the protein. ${ }^{c}$ The side chain of Leu50 is too mobile to be modeled in the WL59 mutant, indicating significant disorder.

single stabilizing mutations have been combined to create a superstable multiple mutant for the proteins: barnase (Serrano et al., 1993); GroEL apical domain (Wang et al., 2000); barstar (Schreiber et al., 1994); and p53 (Nikolova et al., 1998).

Biological Significance of Structural Differences between FKBP12 and FKBP12.6 Isoforms. While it is striking that the structural differences between two isoforms of a protein arises from just one residue change out of 18 , there may be significance for the functional differences between FKBP12 and FKBP12.6. FKBP12 associates with the skeletal ryanodine receptor (RyR1), while FKBP12.6 selectively binds the cardiac ryanodine receptor (RyR2) (Carmody et al., 2001; Gaburjakova et al., 2001; Xin et al., 1999). Ryanodine receptors govern the release of $\mathrm{Ca}^{2+}$ from the sarcoplasmic reticulum in skeletal and heart muscle, thus triggering muscle contraction (Gaburjakova et al., 2001). A mutagenesis study identified three residues, Gln31, Asn32, and Phe59, in FKBP12.6 that confer specificity in binding cardiac RyR2 (Xin et al., 1999). Residues 31 and 32 are located on a $\beta$-turn on the protein surface, some $10 \AA$ from Phe 59 and the FK506/rapamycin binding site. In FKBP12 these residues are Glu31 and Asp32, representing a net charge difference of 2 units. It is possible that the charge difference may have a role in receptor binding affinity and association kinetics, or both. But, there is likely to be little structural differences at this site between the two proteins. Given the large structural response to substitution at residue 59 and its proximity to the FK506/rapamycin binding site, this residue most likely has a role in receptor binding.

In FKBP12, Val63 is located on the same face of the helix to Phe59. It has been suggested that in FKBP12.6 an alanine at this position would facilitate the helical shift into the cavity created by substituting tryptophan for phenylalanine (Dei- 


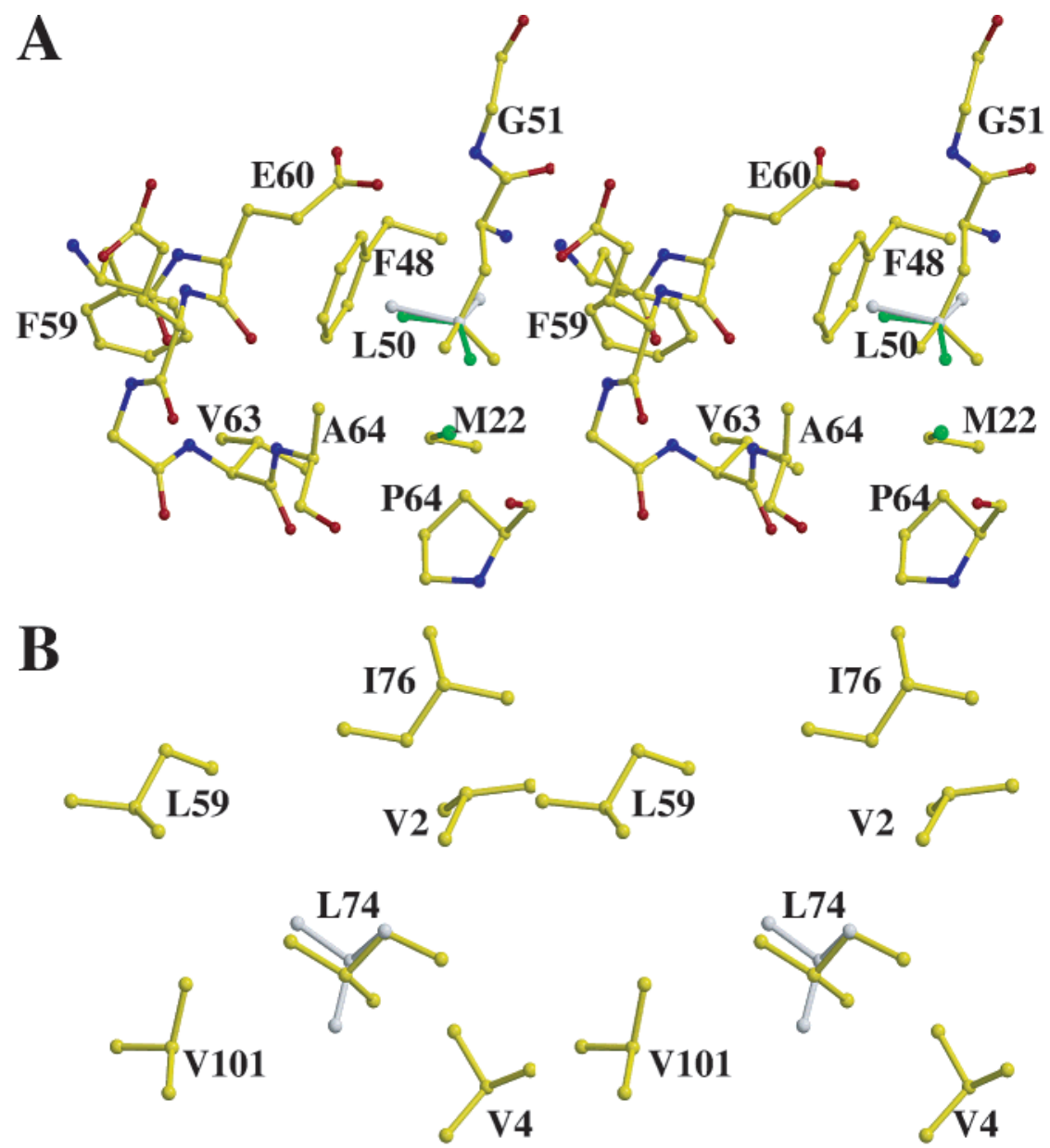

FIGURE 7: Multiple side chain conformations and surrounding residues, shown in stereo. Alternative side chain conformations are shown in white and green. Drawn with MolScript (Kraulis, 1991) and Raster3D (Merrit and Murphy, 1994): (a) Leu50 in the WF59 mutant; (b) Leu74 in the WL59 mutant.

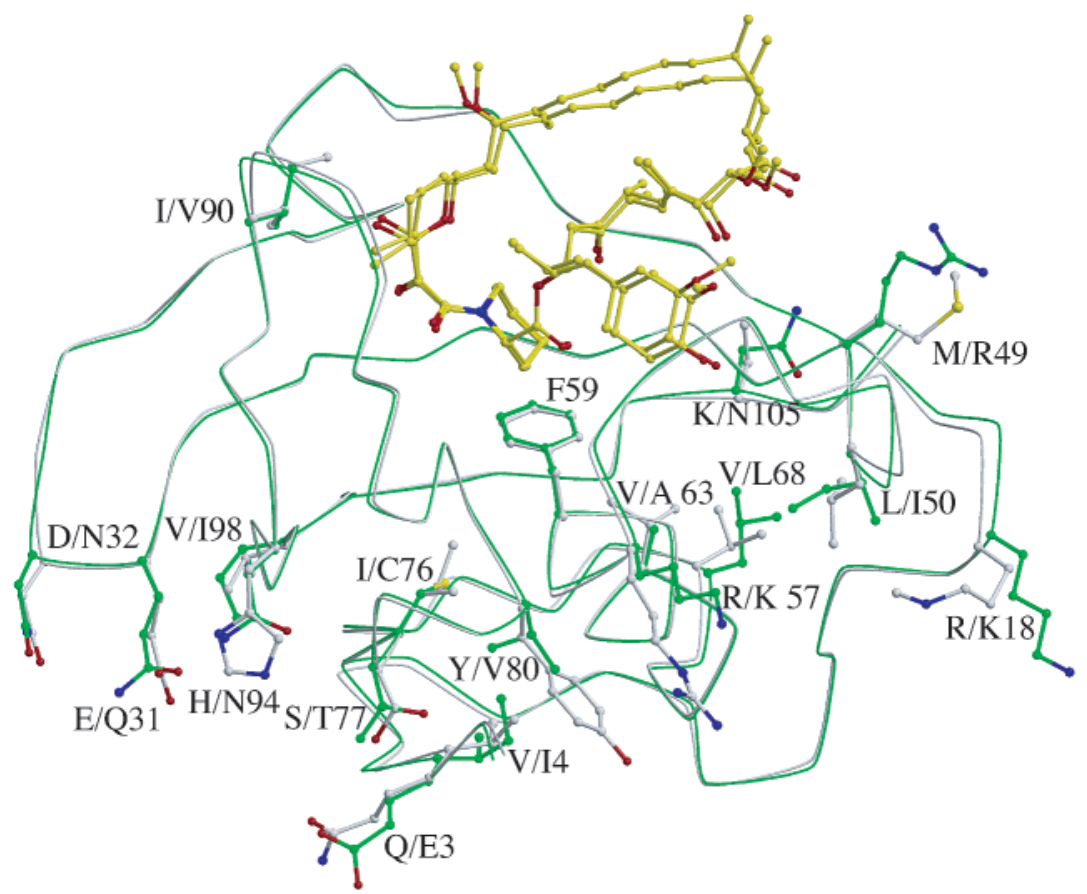

FIGURE 8: Structural overlay of WF59 FKBP12 and wild-type FKBP12.6, showing all 18 residue differences and rapamycin. Drawn with MolScript (Kraulis, 1991) and Raster3D (Merrit and Murphy, 1994).

vanayagam et al., 2000). The results presented here cast some doubt on this, as the shifts seem to be unrelated to all other differences between FKBP12 and FKBP12.6 and due solely to substitution at residue 59. Similarly, differences at residue 90 (Val $\rightarrow$ Ala) at the opposite face of the FK506/rapamycinbinding site to Phe59 are probably too small to have any 


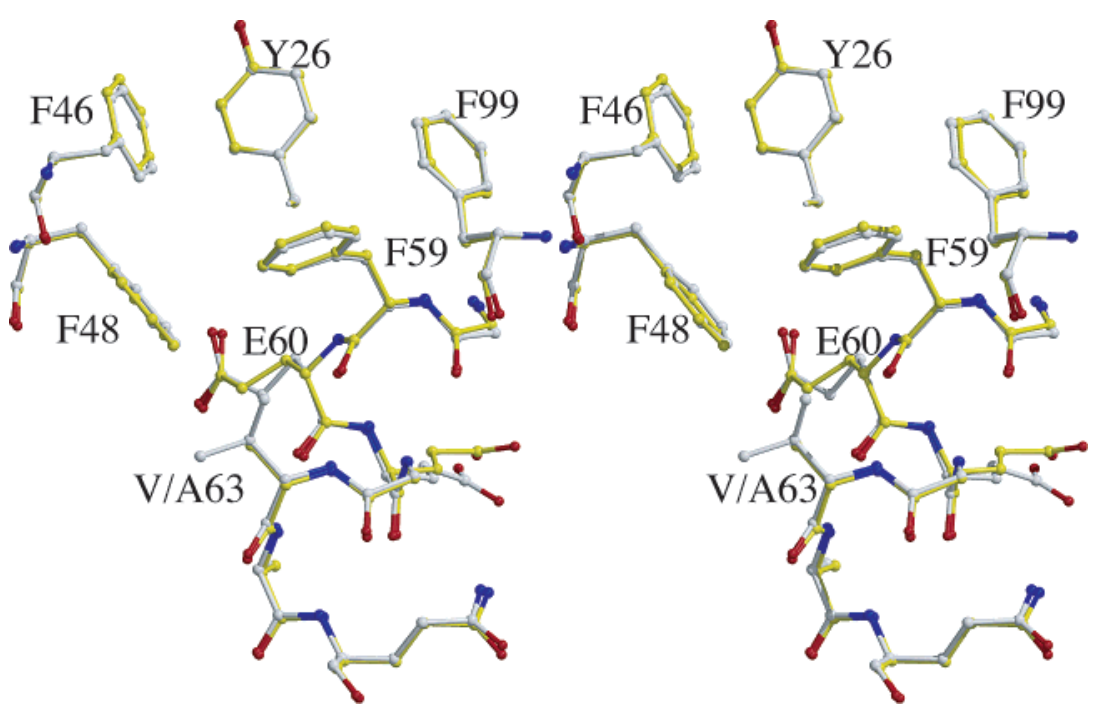

FIGURE 9: Superposition of the structures of WF59 mutant (white bonds) and wild-type FKBP12.6 (yellow bonds), showing the $\alpha$-helix and residues surrounding mutation site. Shown in stereo. Drawn with MolScript (Kraulis, 1991) and Raster3D (Merrit and Murphy, 1994).

significant effect on selective receptor binding. Structural studies of "conservative" mutations Ile $\rightarrow$ Val and Val $\rightarrow$ Ala show, in general very modest structural response to mutation (Buckle et al., 1993; Jackson et al., 1993).

Binding specificity may also arise from sequence variation of RyR: a conserved Val-Pro peptide (LSRLVPLDDLV) in RyR1 forms part of the FKBP12 binding site, whereas the FKBP12.6-binding site of RyR2 consists of a conserved Ile-Pro peptide (LSRLIPLDDLV) (Cameron et al., 1997). In the absence of any structural data for RyR, it is difficult to correlate this subtle sequence difference with the structural differences between FKBP12 and FKBP12.6 caused by variation at residue 59. Residues Gln31 and Asn32 in FKBP12.6 also contribute to specificity in binding cardiac RyR2. The quantitative contribution that each residue makes to binding specificity will be revealed only from binding studies between FKBP variants and the ryanodine receptor.

\section{CONCLUSIONS}

The high-resolution crystal structures of FKBP12 mutants WL49 and WF59 complexed with rapamycin offer several insights into the stability, structure, and function of FKBP12. The increased stability of the mutants is probably due to a combination of improved packing and a favorable entropic gain at the mutation site. The structural differences between FKBP12 and FKBP12.6 can be attributed to residue 59, even though there are 18 amino acid differences in total. Previous work has identified several residues that govern the specificity of binding of FKBP12 and FKBP12.6 for skeletal and cardiac muscle ryanodine receptors. Our findings corroborate the finding that residue 59 plays some part in binding specificity. Further, the combination of structural and thermodynamic analysis show how the protein structure displays a structure-function tradeoff common to many proteins: the presence of Trp59 is required for optimal function, but at the expense of protein stability.

\section{ACKNOWLEDGMENT}

S.E.J. is a Royal Society University Research Fellow. K.F.F. was the recipient of an Elmore Studentship, Gonville and Caius College, Cambridge.

\section{REFERENCES}

1. Atkison, P. (1995) Hypertrophic Cardiomyopathy Associated with Tacrolimus in Paediatric Transplant Patients, Lancet 345.

2. Brillantes, A. B., Ondrias, K., Scott, A., Kobrinsky, E., Ondriasová, E., Moschella, M. C., Jayaraman, T., Landers, M., Ehrlich, B. E., and Marks, A. R. (1994) Stabilization of Calcium Release Channel (ryanodine receptor) Function by FK506-binding protein, Cell 77, 513-523.

3. Brunger, A. T. (1992a) Free R Value: a Novel Statistical Quantity for Assessing the Accuraacy of Crystal Structures, Nature 355, $472-475$

4. Brunger, A. T. (1992b) XPLOR Manual, Version 3.1., Yale University, New Haven, CT.

5. Brunger, A. T. (1993) Assessment of Phase Accuracy by Cross Validation: the Free R Value. Methods and Applications, Acta Crystallogr. D49, 24-36.

6. Buckle, A. M., Henrick, K., and Fersht, A. R. (1993) Crystal Structural Analysis of Mutations in the Hydrophobic Cores of Barnase, J. Mol. Biol. 234, 847-860.

7. Cameron, A. M., Nucifora, F. C. J., Fung, E. T., Livingston, D. J., Aldape, R. A., Ross, C. A., and Snyder, S. H. (1997) FKBP12 binds the Inositol 1,4,5-trisphosphate Receptor at Leucine-Proline (1400-1401) and Anchors Calcineurin to this FK506-like Domain, J. Biol. Chem. 272, 27582-27588.

8. Carmody, M., Mackrill, J. J., Sorrentino, V., and O'Neill, C. (2001) FKBP12 Associates Tightly with the Skeletal Muscle Type 1 Ryanodine Receptor, but not with Intracellular Calcium Release Channels, FEBS Lett. 505, 97-102.

9. CCP4. (1994) The CCP4 Suite: Programs for Protein Crystallography, Acta Crystallogr. D50, 760-763.

10. DeCenzo, M. T., Park, S. T., Jarrett, B. P., Aldape, R. A., Futer, O., Murcko, M. A., and Livingston, D. J. (1996) FK506-binding Protein Mutational Analysis: Defining the Active-site Residue Contributions to Catalysis and the Stability of Ligand Complexes, Protein Eng. 9, 173-180.

11. Deivanayagam, C. C. S., Carson, M., Thotakura, A., Narayana, S. V. L., and Chodavarapu, R. S. (2000) Structure of FKBP12.6 Complex with Rapamycin, Acta Crystallogr. D56.

12. Fulton, K. F., Main, E. R., Daggett, V., and Jackson, S. E. (1999) Mapping the Interactions Present in the Transition State for Unfolding/Folding of FKBP12, J. Mol. Biol. 291, 445-461.

13. Gaburjakova, M., Gaburjakova, J., Reiken, S., Huang, F., Marx, S. O., Rosemblit, N., and Marks, A. R. (2001) FKBP12 Binding Modulates Ryanodine Receptor Channel Gating, J. Biol. Chem. 276, 16931-16935.

14. Harding, M. W., Galat, A., Uehling, D. E., and Schreiber, S. L. (1989) A Receptor for the Immunosuppressant FK506 is a cistrans Peptidyl-Prolyl Isomerase, Nature 341, 758-760.

15. Harrar, Y., Bellini, C., and Faure, J. D. (2001) FKBPs: At the Crossroads of Folding and Transduction, Trends Plant Sci. 6, 426-431. 
16. Jackson, S. E., Moracci, M., Elmasry, N., Johnson, C. M., and Fersht, A. R. (1993a) Effect of Cavity-Creating Mutations in the Hydrophobic Core of Chymotrypsin Inhibitor-2, Biochemistry 32 , 11259-11269.

17. Jayaraman, T., Brillantes, A. M., Timerman, A. P., Fleischer, S., Erdjument-Bromage, H., Tempst, P., and Marks, A. R. (1992) FK506 Binding Protein associated with the Calcium Release Channel (ryanodine receptor), J. Biol. Chem. 267, 9474-9477.

18. Jones, T. A., Zou, J. Y., Cowan, S. W., and Kjeldgaard, M. (1991) Improved Methods for Building Protein Models in Electron Density Maps and the Location of Errors in these Models, Acta Crystallogr. A47, 110-119.

19. Kleywegt, G. J., and Brunger, A. T. (1996) Checking your imagination: applications of the free $R$ value, Structure 4, 897-904.

20. Kraulis, P. (1991) J. Appl. Crystallogr. 24, 946-950.

21. Laitinen, P. J., Brown, K. M., Piippo, K., Swan, H., Devaney, J. M., Brahmbhatt, B., Donarum, E. A., Marino, M., Tiso, N., Viitasalo, M., (2001) Mutations in the Cardiac Ryanodine receptor (RyR2) Gene in Familial Polymorpic Ventricular Tachycardia, Circulation 103, 485-490.

22. Main, E. R., Fulton, K. F., and Jackson, S. E. (1998) ContextDependent Nature of Destabilizing Mutations on the Stability of FKBP12, Biochemistry 37, 6145-6153.

23. Main, E. R., Fulton, K. F., and Jackson, S. E. (1999a) Folding Pathway of FKBP12 and Characterisation of the Transition State, J. Mol. Biol. 291, 429-444.

24. Main, E. R., and Jackson, S. E. (1999b) Does Trifluoroethanol Affect Folding Pathways and can it be Used as a Probe of Structure in Transition States? Nat. Struct. Biol. 6, 831-835.

25. Main, E. R. G., Fulton, K. F., Daggett, V., and Jackson, S. E. (2001) A Comparison of Experimental and Computational Methods for Mapping the Interactions Present in the Transition State for Folding of FKBP12, J. Biol. Phys. 27, 99-117.

26. Marks, A. R. (2002) Ryanodine Receptors, FKBP12, and Heart Failure, Frontiers Biosci. 7, 1-11.

27. McPherson, A. (1982) The Preparation and Analysis of Protein Crystals, John Wiley \& Sons, New York.

28. Merrit, E. A., and Murphy, M. E. P. (1994) Acta Crystallogr. D50, 869-873.

29. Meiering, E. M., Serrano, L., and Fersht, A. R. (1992) Effect of Active Site Residues in Barnase on Activity and Stability, J. Mol. Biol. 225, 585-589.

30. Michnick, S. W., Rosen, M. K., Wandless, T. J., Karplus, M., and Schreiber, S. L. (1991) A High Resolution Structure of FKBP, a Rotamase and Receptor for FK506 and Rapamycin, Science 252, 836-842.

31. Moore, J. M., Peattie, D. A., Fitzgibbon, M. J., and Thomson, J. A. (1991) Solution Structure of the Major Binding Protein for the Immunosuppressant FK506, Science 351, 248-250.

32. Morris, A. L., Macarthur, M. W., Hutchinson, E. G., and Thornton, J. M. (1992) Stereochemical Quality of Protein Structure Coordinates, Proteins 12, 345-364.

33. Murshudov, G. N., Vagin, A. A., and Dodson, E. J. (1997) Refinement of Macromolecular Structures by the Maximumlikelihood, Methodol. Acta Crystallogr. D53, 240-255.
34. Nikolova, P. V., Henckel, J., Lane, D. P., and Fersht, A. R. (1998) Semirational Design of Active Tumor Suppressor p53 DNA Binding Domain with Enhanced Stability, Proc. Natl. Acad. Sci. U.S.A. 95, 14675-14680.

35. Ponder, J. W., and Richards, F. M. (1987) Tertiary templates for proteins. Use of Packing Criteria in the Enumeration of allowed Sequences for Different Structural Classes, J. Mol. Biol. 193, 775791.

36. Priori, S. G., Napolitano, C., Tiso, N., Memmi, M., Vignati, G., Bloise, R., Sorrentino, V., and Danieli, G. A. (2001) Mutations in the Cardiac Ryanodine Receptor Gene ( $h R Y R 2)$ Underlie Catecholaminergic Polymorphic Ventricular Tachycardia, Circulation 103, 196-200.

37. Read, R. J. (1986) Improved Fourier coefficients for Maps using Phases from Partial Structures with Errors, Acta Crystallogr. A42, 140-149.

38. Schreiber, C., Buckle, A. M., and Fersht, A. R. (1994) Stability and Function: Two Constraints in the Evolution of Barstar and other Proteins, Structure 2, 945-951.

39. Schreiber, S. L. (1991) Chemistry and Biology of the Immunophilins and Their Immunosuppressant Ligands, Science 251, 283287.

40. Serrano, L., Day, A. G., and Fersht, A. R. (1993) Stepwise Mutation of Barnase to Binase. A Procedure for Engineering increased Stability of Proteins and an Experimental Analysis of the Evolution of Protein Stability, J. Mol. Biol. 233, 305-312.

41. Shou, W., Aghdasi, B., Armstrong, D. L., Guo, Q., Bao, S., Charng, M. J., Mathews, L. M., Schneider, M. D., Hamilton, S. L., and Matzuk, M. M. (1998) Cardiac Defects and Altered Ryanodine Receptor Function in Mice Lacking FKBP12, Nature 391, 489-492.

42. Timerman, A. P., Onoue, H., Xin, H. B., Barg, S., Copello, J., Wiederrecht, G., and Fleischer, S. (1996) Selective Binding of FKBP12.6 by the Cardiac Ryanodine Receptor, J. Biol. Chem. 271, 20385-20391.

43. van Duyne, G. D., Standaert, R. F., Karplus, P. A., Schreiber, S. L., and Clardy, J. (1991a) Atomic Structure of FKBP-FK506, an Immunophilin-Immunosuppressant Complex, Science 252.

44. van Duyne, G. D., Standaert, R. F., Schreiber, S. L., and Clardy, J. (1991b) Atomic Structure of the Rapamycin Human Immunophilin FKBP-12 Complex, J. Am. Chem Soc. 113, 7433-7434.

45. van Duyne, G. D., Standaert, R. F., Karplus, P. A., Schreiber, S. L., and Clardy, J. (1993) Atomic structures of the Human Immunophilin FKBP12 Complexes with FK506 and Rapamycin, J. Mol. Biol. 229, 105-124.

46. Wang, Q., Buckle, A. M., and Fersht, A. R. (2000) Stabilization of GroEl Minichaperones by Core and Surface Mutations, J. Mol. Biol. 298, 917-926.

47. Xin, H. B., Rogers, K., Qi, Y., Kanematsu, T., and Fleischer, S. (1999) Three Amino Acid Residues determine Selective Binding of FK506-binding Protein 12.6 to the Cardiac Ryanodine Receptor, J. Biol. Chem. 274, 15315-15319.

BI020564A 